\title{
What Is an Intracluster Correlation Coefficient? Crucial Concepts for Primary Care Researchers
}

\author{
Shersten Killip, $M D, M P H^{1}$ \\ Ziyad Mabfoud, $P b D^{2}$ \\ Kevin Pearce, $M D, M P H^{1}$ \\ 'Department of Family Practice and \\ Community Medicine, University of \\ Kentucky, Lexington, Ky \\ ${ }^{2}$ Department of Statistics, University \\ of Kentucky, Lexington, Ky
}

Conflict of interest: none reported

\section{CORRESPONDING AUTHOR}

Shersten Killip, MD, MPH

K-302 Kentucky Clinic 0284

$740 \mathrm{~S}$. Limestone

Lexington, KY 40536-0284

skill2@email.uky.edu

\begin{abstract}
BACKGROUND Primary care research often involves clustered samples in which subjects are randomized at a group level but analyzed at an individual level. Analyses that do not take this clustering into account may report significance where none exists. This article explores the causes, consequences, and implications of cluster data.
\end{abstract}

METHODS Using a case study with accompanying equations, we show that clustered samples are not as statistically efficient as simple random samples.

RESULTS Similarity among subjects within preexisting groups or clusters reduces the variability of responses in a clustered sample, which erodes the power to detect true differences between study arms. This similarity is expressed by the intracluster correlation coefficient, or $\rho$ (rho), which compares the within-group variance with the between-group variance. Rho is used in equations along with the cluster size and the number of clusters to calculate the effective sample size (ESS) in a clustered design. The ESS should be used to calculate power in the design phase of a clustered study. Appropriate accounting for similarities among subjects in a cluster almost always results in a net loss of power, requiring increased total subject recruitment. Increasing the number of clusters enhances power more efficiently than does increasing the number of subjects within a cluster.

CONCLUSIONS Primary care research frequently uses clustered designs, whether consciously or unconsciously. Researchers must recognize and understand the implications of clusters to avoid costly sample size errors.

Ann Fam Med 2004;2:204-208. DOI: 10.1370/afm.141.

\section{INTRODUCTION}

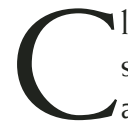
lustered samples are not as statistically efficient as simple random samples. Similarities among subjects in clusters can reduce the variability of responses from a cluster compared with those expected from a simple random sample. If statistics meant for simple random samples are used to design and analyze clustered studies, they will result in overestimation of the effective sample size. This issue is important for primary care research, because the design of many primary care research studies creates clusters.

This article will use a case study to introduce the concepts involved in cluster sampling. It is intended as an introduction to the concepts and language of cluster sampling; researchers are encouraged to consult a statistician familiar with cluster sampling to help in the design and analysis phases of clustered studies. The goal is to raise the awareness of cluster sampling issues among primary care researchers and to help primary care researchers design and publish statistically rigorous findings. 


\section{DEFINITION AND EXPLANATION OF CLUSTERED DESIGNS}

\section{Case Study}

A clinical trial was designed to evaluate the impact of physician advice on condom use. The outcome was patient-reported use of condoms 6 months after randomization to a control group or a counseling group. The investigator avoided contamination by randomizing the physicians to be control physicians or counseling physicians (randomizing at the physician level) but wanted to analyze data at the patient level. Four physicians in solo practice were recruited, 2 to counsel, and 2 to be controls. A sample-size calculation was done, which suggested that for a 2 -sample $t$ test, a minimum effect size of 0.5 , and a significance level of $.05,128$ patients total would be necessary to achieve a power of $80 \%$. Each physician was therefore asked to recruit 32 patients. When the paper was submitted to a journal, the paper was rejected for "erroneous statistics" and "inadequate power: $61 \%$.

What happened? Most statistical methodologies were designed to analyze data that is both selected and analyzed on the same level. Clustered data result when some preexisting group structure is used to select study participants, but the researcher is interested in the individual level data. Clustered designs can be used for many reasons, but they always cause some loss of statistical efficiency as a result of the "relatedness" within the preexisting groups. Primary care research, which often studies patients from multiple private practices, can produce clustered results by selecting groups of patients at the practice (or practitioner) level, then analyzing the data at the individual patient level, as in our case study.

Why does clustering erode statistical power? Consider the nature of preexisting groups. Most groups form because of some kind of selection factors. Among patients who all see the same primary care physician, there can be many similarities that may include geographic, socioeconomic, racial, ethnic, sexual, religious, political, or age-related similarities, stemming from the propensity of patients to choose a physician with whom they identify. All of these factors can have some impact on the average response of one physician's patients compared with another's.

The responses of persons selected by any or all of the factors mentioned above tend to be more similar to one another's than the responses of a group of individuals selected truly at random. Because these responses are similar, they lead to a decrease in the variation among responses of persons in the same cluster, or the variance of the within-cluster responses. This similarity among responses within a group can magnify the apparent differences in outcomes or responses between groups, and they must be taken into account. Adjustment for clustering thus results in a reduction of the effective sample size.

In the case study, solo practitioners were chosen at the physician level to keep the discussion simple. Figure 1 illustrates the design of the study. Physicians who choose to work together, however, share similarities just as their patients do, and these similarities must be taken into account. Using physicians who worked together would have introduced a third level, the practice level, to our study. This concept is illustrated in Figure 2. Multilevel clustering is termed nesting, and there are specific statistics to deal with that mathematical situation. Clustering is a specific term for the simplest type of nesting, using only 2 levels of data, as shown in Figure 1.

\section{ADVANTAGES AND DISADVANTAGES OF CLUSTERED DESIGNS}

While the loss of statistical efficiency and the need to recruit more study participants are clear disadvantages of clustered studies, there are some advantages to clustering. Clustering is often used for practical reasons when a simple random sample would be unrealistic. For example, a random survey of all patients in a given area would be extremely difficult. A clustered survey of randomly chosen patients within primary care practices is much more practical.

\section{Figure 1. Two-level nesting, or clustering.}

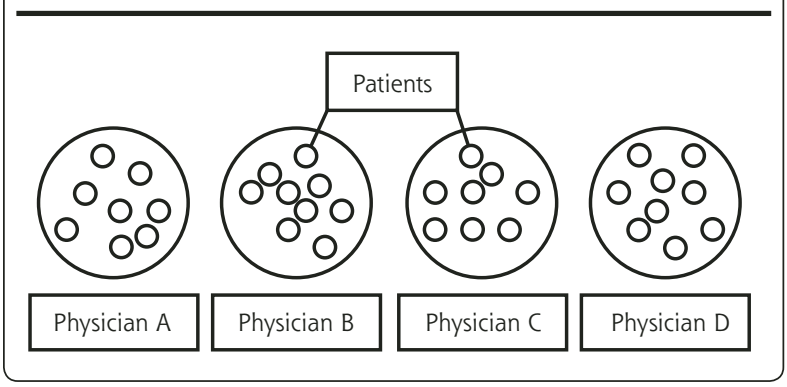

Figure 2. Three-level nesting.

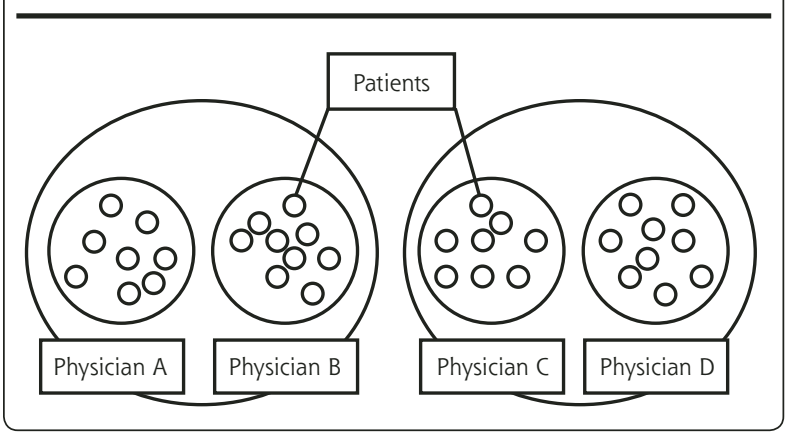


Clustering is the design of choice to avoid a phenomenon known as contamination. In our example, asking the same physician to present 2 entirely different counseling scripts to his or her patients is impractical ${ }_{i}$ the physician will likely get confused. Also, patients of the same physician may be acquainted. Some patients could pass on a version of the counseling to other patients. As a result, the loss of efficiency from clustering is necessary to preserve the integrity of the intervention

\section{THE INTRACLUSTER CORRELATION COEFFICIENT, OR $\rho$}

The intracluster correlation coefficient (ICC), or $\rho$ (the Greek rho), is a measure of the relatedness of clustered data. It accounts for the relatedness of clustered data by comparing the variance within clusters with the variance between clusters. Mathematically, it is the between-cluster variability divided by the sum of the within-cluster and between-cluster variabilities.

Equation $1:^{*}$

$$
\text { ICC or } \rho=\frac{S_{b}^{2}}{\left(S_{b}^{2}+S_{w}^{2}\right)},
$$

where $s_{b}^{2}=$ the variance between clusters, and $s_{w}^{2}=$ the variance within clusters.

Values of $\rho$ range from 0 to 1 in human studies. From equation 1 , as the within-cluster variance $\left(s_{w}^{2}\right)$ moves toward $0, \rho$ gets closer and closer to 1 . In the theoretical case where $\rho=1$, all responses within a cluster are identical. In that case the effective sample size is reduced to the number of clusters.

A very small value for $\rho$ implies that the withincluster variance is much greater than the betweencluster variance, and a $\rho$ of 0 shows that there is no correlation of responses within a cluster. Usually, values of $r$ are between 0.01 and 0.02 in human studies. ${ }^{2-4}$ The calculation of $\rho$ usually requires a pilot study. We encourage all investigators to publish their $\rho$ values, which will (eventually) aid in being able to estimate $\rho$ for a given type of population.

\section{EFFECTIVE SAMPLE SIZE AND THE DESIGN EFFECT}

In accounting for the similarities among clustered subjects, there is a net loss of independent data. The effective sample size is the term used to describe the sample size in clustered samples compared with the number

*For equal cluster size, a weighted average is needed to adjust this formula. of subjects actually enrolled in the study. For example, if you have 4 physicians' offices (from the case study above) enrolling 32 patients each, you have 128 subjects in your study. Depending on the intracluster correlation coefficient and the design effect, however, you may effectively have far fewer subjects enrolled in your trial from a statistical perspective.

To get the effective sample size, the total sample size (the number of patients per cluster times the number of clusters) is divided by a correction factor that includes $\rho$ and the sample size per cluster ( $\mathrm{m})$. This correction factor is called the design effect. In the case study above, we created the special case of clustered data with all groups having the same number of subjects (each physician recruited 32 patients). In this special case:

Equation 2:

$$
\mathrm{ESS}=\frac{\mathrm{mk}}{\mathrm{DE}}
$$

and equation 3 :

$$
\mathrm{DE}=1+\rho(\mathrm{m}-1),
$$

where $\mathrm{m}=$ number of subjects in a cluster, $\mathrm{k}=$ number of clusters, $\mathrm{mk}=$ total number of subjects in a clustered study, ESS = effective sample size, $\mathrm{DE}=$ design effect, and $\rho=$ intracluster correlation coefficient (see equation 1).

If $\rho=0$, then the design effect $=1$, and the sample size is unaffected. If $\rho>0$, even if it is still very small, the design effect may be magnified by a large cluster size (m). This would then reduce the effective sample size of the study (see equation 2). If $\rho=1$, the design effect (equation 2) is 1 , and the effective sample size therefore reduces to $k$, the number of clusters.

These equations can be reversed in the planning phase to calculate correctly the total sample size needed for a clustered study. All power calculations and resultant sample size estimates can be calculated initially using usual formulas for a clustered study, which will give researchers the effective sample size. Equation 2 can be used to find $\mathrm{mk}$, or the total required sample size, given the effective sample size and design effect

\section{THE EFFECT OF $\rho$ AND THE DESIGN EFFECT ON POWER AND SAMPLE SIZE CALCULATIONS}

To illustrate the effect of $\rho$ and the design effect on sample size and power, we will do a sample calculation. Using our case study, we have 4 physicians 


\section{Table 1. Effective Sample Size and Power Holding mk Constant}

\begin{tabular}{|c|c|c|c|c|c|}
\hline \multirow{2}{*}{$\begin{array}{c}\text { Number of } \\
\text { Physicians } \\
\text { k }\end{array}$} & \multirow{2}{*}{$\begin{array}{c}\text { Number of } \\
\text { Patients } \\
\text { m }\end{array}$} & \multirow{2}{*}{$\begin{array}{c}\text { Total } \\
\text { Number } \\
\text { (mk) }\end{array}$} & \multicolumn{2}{|c|}{$\rho=0.017$} & \multirow{2}{*}{$\begin{array}{l}\text { Power } \\
t \text { Test* }\end{array}$} \\
\hline & & & $\mathrm{DE}$ & ESS & \\
\hline 4 & 32 & 128 & 1.527 & 84 & 61 \\
\hline 8 & 16 & 128 & 1.255 & 102 & 70 \\
\hline 16 & 8 & 128 & 1.119 & 114 & 75 \\
\hline 32 & 4 & 128 & 1.051 & 122 & 78 \\
\hline 64 & 2 & 128 & 1.017 & 126 & 79 \\
\hline 128 & 1 & 128 & 1.000 & 128 & 80 \\
\hline \multicolumn{6}{|c|}{$\begin{array}{l}\text { *Power to detect a minimum effect size of } 0.5 \text { when one half the clusters is randomly assigned to treatment and } \\
\text { the other half to placebo, and mean responses are compared using a } 2 \text {-sample } t \text { test made at the } .05 \text { level of } \\
\text { significance. } \\
m=\text { number of subjects in a cluster; } k=\text { number of clusters; } \mathrm{mk}=\text { total number of subjects in a clustered study; } \\
\mathrm{DE}=\text { design effect; and ESS = effective sample size. }\end{array}$} \\
\hline
\end{tabular}

\section{Table 2. Effective Sample Size and Power Holding k Constant}

\begin{tabular}{|c|c|c|c|c|c|}
\hline \multirow{2}{*}{$\begin{array}{c}\text { Number of } \\
\text { Physicians } \\
\text { k }\end{array}$} & \multirow{2}{*}{$\begin{array}{c}\text { Number } \\
\text { of Patients } \\
\mathrm{m}\end{array}$} & \multirow{2}{*}{$\begin{array}{c}\text { Total } \\
\text { Number } \\
\text { (mk) }\end{array}$} & \multicolumn{2}{|c|}{$\rho=0.017$} & \multirow{2}{*}{$\begin{array}{l}\text { Power } \\
t \text { Test* }\end{array}$} \\
\hline & & & $\mathrm{DE}$ & ESS & \\
\hline 4 & 10 & 40 & 1.153 & 34 & 29 \\
\hline 4 & 20 & 80 & 1.323 & 60 & 47 \\
\hline 4 & 40 & 160 & 1.663 & 96 & 67 \\
\hline 4 & 80 & 320 & 2.343 & 136 & 82 \\
\hline
\end{tabular}

* Power to detect a minimum effect size of 0.5 when one half the clusters is randomly assigned to treatment and the other half to placebo, and mean responses are compared using a 2 -sample $t$ test made at the .05 level of significance.

$\mathrm{m}=$ number of subjects in a cluster; $\mathrm{k}=$ number of clusters; $\mathrm{mk}=$ total number of subjects in a clustered study; $\mathrm{DE}=$ design effect; and ESS = effective sample size.

\section{Table 3. Effective Sample Size and Power Holding m Constant}

\begin{tabular}{|c|c|c|c|c|c|}
\hline \multirow{2}{*}{$\begin{array}{c}\text { Number of } \\
\text { Physicians } \\
\text { k }\end{array}$} & \multirow{2}{*}{$\begin{array}{c}\text { Number } \\
\text { of Patients } \\
\text { m }\end{array}$} & \multirow{2}{*}{$\begin{array}{c}\text { Total } \\
\text { Number } \\
\text { (mk) }\end{array}$} & \multicolumn{2}{|c|}{$\rho=0.017$} & \multirow{2}{*}{$\begin{array}{l}\text { Power } \\
t \text { Test* }\end{array}$} \\
\hline & & & $\mathrm{DE}$ & ESS & \\
\hline 2 & 10 & 20 & 1.153 & 18 & 16 \\
\hline 4 & 10 & 40 & 1.153 & 36 & 30 \\
\hline 8 & 10 & 80 & 1.153 & 70 & 50 \\
\hline 16 & 10 & 160 & 1.153 & 138 & 83 \\
\hline \multicolumn{6}{|c|}{$\begin{array}{l}\text { *Power to detect a minimum effect size of } 0.5 \text { when one half the clusters is randomly assigned to treatment and } \\
\text { the other half to placebo, and mean responses are compared using a } 2 \text {-sample } t \text { test made at the } .05 \text { level of } \\
\text { significance. } \\
m=\text { number of subjects in a cluster; } k=\text { number of clusters; } m k=\text { total number of subjects in a clustered study; } \\
D E=\text { design effect; and ESS = effective sample size. }\end{array}$} \\
\hline
\end{tabular}

Note that despite the small value for $\rho$, the design effect came out to 1.527 . This reduced our effective sample size to 84 compared with the 128 subjects actually enrolled in the trial, which explains why the power was only $61 \%$.

If we change the numbers for $\mathrm{m}$ and $\mathrm{k}$, we can show that the magnitude of the design effect is highly dependent on $\mathrm{m}$, the number of patients in a cluster. Table 1 illustrates the changes in effective sample size and power for our example as we vary $m$ and $k$ but hold the product mk constant. Table 2 shows the effect of increasing $\mathrm{m}$ while holding k constant. Note the increasing design effect as $m$ increases and its effect on the effective sample size i $_{\text {the }}$ investigator would have needed to recruit almost 80 patients per physician (320 total subjects) to adequately power his study with only 4 physicians. Table 3 shows that by increasing the number of physicians he enrolled in his study to 16 , he would only have needed a total of 160 subjects to reach $80 \%$ power.

\section{SUMMARY}

The intracluster correlation coefficient, or $\rho$, is a measure of relatedness of responses within a cluster. In human studies it is usually small, but in the design effect it is magnified by the number of elements in the cluster $(\mathrm{m})$. The smaller the design effect, the larger the effective sample size. A high $\mathrm{k}$ (number of clusters) and

recruiting 32 patients each. Let us say that $\rho=0.017$ in this case. What is the effective sample size after adjusting for clustering?

$$
\begin{aligned}
& \text { If } \mathrm{m}=32, \mathrm{k}=4 \text {, and } \rho=0.017 \\
& \text { ESS }=\frac{\mathrm{mk}}{1+\rho(\mathrm{m}-1)}=\frac{(32)(4)}{1+0.017(32-1)}=\frac{128}{1.527}=84
\end{aligned}
$$

a low $\mathrm{m}$ (number of elements within a cluster) give the smallest design effect. When designing studies, increasing clusters $(\mathrm{k})$ will increase the study's power more than increasing the elements in the clusters $(\mathrm{m})$. Standard formulas can be used to calculate sample sizes in clustered situations, but the resulting effective sample size (ESS) must then be adjusted using the design effect (DE) to find the total required sample size. 
To read or post commentaries in response to this article, see it online at http://www.annfammed.org/cgi/content/full/2/3/204.

Key words: Statistics; cluster analysis; data interpretation, research design; primary care; practice-based research; methods/quantitative; theory

Submitted August 18, 2003; submitted, revised, December 29, 2003; accepted January 20, 2004.

Funding support: This work was supported by grant \# 1 D14 HP 00041 from the Health Resources and Services Administration.

Previous presentations: This work was presented by Dr. Shersten Kil lip on December 6, 2002, at the Primary Care Research Methods and Statistics Conference in San Antonio, Tex, and on March 22, 2003, at the 2003 Convocation of Practices (hosted by the AAFP National Network for Family Practice and Primary Care Research and the Federation of PracticeBased Research Networks) in Arlington, Va. Both presentations were under the title "What Is an Intracluster Correlation Coefficient? Crucial Concepts for Novice PBRN Researchers."

\section{References}

1. Donner A, Klar N. Design and Analysis of Cluster Randomization Trials in Health Research. American ed. New York, NY: Oxford University Press; 2000:9,112-113.

2. Murray DM, Rooney BL, Hannan PJ, et al. Intraclass correlation among common measures of adolescent smoking. Am J Epidemiol. 1992; 140:1038-1050.

3. Murray DM, Short BJ. Intraclass correlation among measures related to alcohol use by young adults. J Studies Alcohol. 1995;56: 681-694.

4. Murray DM, Short BJ. Intraclass correlation among measures related to alcohol use by adolescents Add Behav. 1997;22:1-12.

\section{For Further Reading:}

1. Donner A, Klar N. Design and Analysis of Cluster Randomization Trials in Health Research. American ed. New York, NY: Oxford University Press; 2000. [Entire book.]

2. Cochran WG. Sampling Techniques. New York, NY: John Wiley and Sons; 1977. 